\title{
Curcumin improves hippocampal function in healthy older adults: a three month randomised controlled trial
}

\author{
Andrew Scholey, Katherine Cox, Andrew Pipingas and David White \\ Swinburne University, Melbourne, Australia
}

\section{Abstract}

The flavonoid curcumin is believed to be responsible for the purported health benefits of turmeric. Like other flavonoids, curcumin affects several systemic and central processes involved in neurocognitive aging. We have previously shown that one month administration of a highly bioavailable curcumin extract (Longvida ${ }^{\mathrm{TM}}$ ) improved working memory and reduced fatigue and workload stress in an older, cognitively intact cohort ${ }^{(1)}$. This study focused on the effects of the same extract, focusing on memory tasks subserved by the hippocampus, one of two areas of the adult brain believed to be capable of adult neurogenesis.

Eighty healthy older participants (aged 50-80 years, mean $=68.1, \pm$ SD 6.34 ) took part in this double-blind, placebo-controlled, parallel-groups trial. Volunteers were randomised to receive administration of $400 \mathrm{mg}$ daily Longvida ${ }^{\mathrm{TM}}$ (containing $80 \mathrm{mg}$ curcumin) or a matching placebo. Assessment took place at baseline and 4 and 12 weeks thereafter. Outcomes included two tasks evaluating memory processes relevant to hippocampal function. These were i) a human analogue of the widely used rodent Morris Water Maze - the virtual Morris Water Maze (vMWM) and ii) a Mnemonic Similarity task evaluating pattern separation. Measures of mood, cardiovascular function and other blood biomarkers were collected, and a subset of the cohort underwent neuroimaging using functional magnetic resonance imaging.

Compared with placebo, there were a number of improvements in the curcumin group. The curcumin group had significantly better performance at 12 weeks on the virtual Morris Water Maze $(\mathrm{p}=.019)$. Curcumin was also associated with better performance on a pattern separation task $(\mathrm{p}=.025)$. Curcumin was also associated with number of significantly benefits to mood, including, from the Profile of Mood States (POMS), including, at 28 days only, total mood disturbance $(p=.006)$, tension-anxiety $(p=.028)$, confusionbewilderment $(p=.019)$, anger-Hostility $(p=.009)$. There were also significant benefits to the POMS fatigue scores at both assessments $(\mathrm{p} \leq .011)$. There were no group differences in biomarker levels.

These results confirm that Longvida ${ }^{\mathrm{TM}}$ curcumin improves aspects of mood and working memory in a healthy older cohort. The pattern of results is consistent with improvements in hippocampal function and may hold promise for alleviating cognitive decline in populations at risk of pathological cognitive decline.

\section{Conflict of Interest}

This study was funded by a grant from Verdure Sciences. Scholey has received research grants, consultancy payments, travel funding and speakers fees from the nutrition industry.

\section{Reference}

1. Cox C, Pipingas A, Scholey A (2015) Investigation of the effects of solid lipid curcumin on cognition and mood in a healthy older population. Journal of Psychopharmacology 29, 642-651. 